\title{
Colonoscopic allergen provocation (COLAP): a new diagnostic approach for gastrointestinal food allergy
} S C Bischoff, J Mayer, J Wedemeyer, P N Meier, G Zeck-Kapp, B Wedi, A Kapp, Y Cetin,
M Gebel, M P Manns

\begin{abstract}
Background-The clinical relevance of gastrointestinal food allergy in adults is largely unknown because the mechanisms are poorly understood and the diagnosis is difficult to confirm.

Aims-To improve the diagnostic means for confirming intestinal food allergy on an objective basis, a new colonoscopic allergen provocation (COLAP) test was developed.
\end{abstract}

Patients-The COLAP test was performed in 70 adult patients with abdominal symptoms suspected to be related to food allergy, and in five healthy volunteers.

Methods-During the COLAP test, the caecal mucosa was challenged endoscopically with three food antigen extracts, a buffer control, and a positive control (histamine). The mucosal weal and flare reaction was registered semiquantitatively 20 minutes after challenge, and tissue biopsy specimens were examined for mast cell and eosinophil activation.

Results-No severe systemic anaphylactic reactions were found in response to intestinal challenge. The COLAP test was positive to at least one food antigen in 54 of 70 patients $(77 \%)$, whereas no reaction in response to antigen was found in healthy volunteers. Antigen induced weal and flare reactions were correlated with intestinal mast cell and eosinophil activation, as well as with patients' history of adverse reactions to food, but not with serum concentrations of total or specific IgE or skin test results.

Conclusion-The COLAP test may be a useful diagnostic measure in patients with suspected intestinal food allergy and may provide a new tool for the study of underlying mechanisms.

(Gut 1997; 40: 745-753)

Keywords: food allergy, inflammatory bowel disease, mast cells, eosinophils, provocation test.

Department of Anatomy, Medical

School of Hannover, D-30623 Hannover, Germany

Y Cetin

Correspondence to:

Dr S C Bischoff,

Department of

Gastroenterology and

Hepatology, Medical Schoo

of Hannover, D-30623

Hannover, Germany.

Accepted for publication 28 January 1997 a cause of gastrointestinal symptoms has been controversially for many possibly due to the lack of a clear definition of such syndromes, the few epidemiological data available, particularly in adults, and the limited knowledge about underlying mechanisms. ${ }^{1-5}$ In particular, the means to confirm the diagnosis of gastrointestinal food allergy are insufficient at present, and therefore it remains difficult to identify afflicted patients on an objective basis. Laboratory variables indicating gastrointestinal food allergy are lacking and skin tests are known to be of limited value in confirming or excluding the diagnosis. ${ }^{6-8}$ Therefore, we developed a new diagnostic approach for intestinal food allergy, the colonoscopic allergen provocation test (COLAP test). Provocation tests are established for the nasal, conjunctival, and bronchial mucosa, and their value in confirming the diagnosis of allergic disease and to identify allergens of relevance is well recognised. A few attempts have been made to develop similar tests for gastrointestinal mucosa, but they could not be established for clinical practice. ${ }^{9-12}$ However, there is striking evidence that allergic reactions occur in the intestinal tract, which must balance two major and largely exclusive functions - nutritional uptake and host defence. To perform these functions, the intestinal barrier is supplied with specialised epithelial cells and a complex network consisting of immunocompetent cells, inflammatory cells, nerves, and blood vessels. Any dysregulation of the intestinal homeostasis may lead to an increased influx of antigens or an abnormal immune reaction. ${ }^{3}$ Therefore, it may be that in certain people, the intestinal mucosa is predestined for allergic reaction against foreign antigens. The COLAP test, in which the intestinal mucosa is challenged endoscopically with antigen extracts, may allow the identification of patients developing a hypersensitivity reaction of the intestinal mucosa in response to particular antigens.

\section{Methods}

PATIENTS AND CONTROLS

Seventy selected patients with chronic abdominal symptoms and suspected gastrointestinal food allergy (17 to 67 years of age, 27 men, 43 women) and five healthy volunteers (26 to 33 years of age, two men, three women), underwent the COLAP test after written informed consent. Permission to conduct the study was obtained from the local ethics committee of the Medical School of Hannover. Each patient had non-specific abdominal symptoms such as diarrhoea $(66 \%)$, abdominal pain $(47 \%)$, flatulence $(27 \%)$, cramps (24\%), obstipation (13\%), and vomiting $(10 \%)$. Sixty one of the 70 patients had clear food intolerances related to those 
symptoms (13 also presenting with oral allergy syndrome). We also included nine patients with gastrointestinal symptoms who had no clear history of adverse reaction to food, but a history of atopic disease and high concentrations of specific IgE or positive skin tests for particular food antigens suggesting intestinal allergy as a cause for their complaints. Every patient underwent a routine programme of gastrointestinal diagnostics including physical examination, measurement of laboratory variables, endoscopy, and histology. Seventeen of 70 patients had inflammatory bowel conditions (five Crohn's disease, three ulcerative colitis, four eosinophilic enteritis, three coeliac disease, and two indeterminate colitis). Thirty nine patients had a history of atopic disease such as seasonal rhinitis, extrinsic asthma, or atopic dermatitis. Raised serum IgE concentrations $(>100 \mathrm{U} / \mathrm{ml})$ were found in 38 patients. Increased numbers of eosinophils were present in the colonic lamina propria $(>8 \%)$ of 14 patients and in the peripheral blood ( $>10 \%$ of total leucocytes) of six patients. Nine patients had been treated with disodium cromoglycate and five of them improved. For the control group, five healthy subjects with a negative history, normal $\operatorname{IgE}$ concentrations, negative skin tests, and negative radioallergosorbent assay (RAST) against the most common allergens, and no clinical signs of atopy or food intolerance were selected.

\section{COLONOSCOPIC ALLERGEN PROVOCATION (COLAP) TEST}

Allergen provocation was performed in the caecum during colonoscopy (videocoloscope CF100HL, Olympus Optical Company, Hamburg, Germany) provided that the macroscopic aspect of the caecal mucosa was normal. None of the patients were anaesthetised, some received promethazine (the day before the COLAP test) and/or midazolam and pethidine intravenously (just before or during the COLAP test) if necessary. Treatment of patients with prednisolone ( $n=7$; maximal dose $10 \mathrm{mg} /$ day), antihistamines $(\mathrm{n}=3)$, or disodium cromoglycate $(n=9)$ was terminated at least three days before the COLAP test. The caecum was chosen because its peristaltic movements proved to be less pronounced than that of other segments of the large intestine. According to previous studies performed in five subjects, we obtained, compared with the caecum, similar but weaker mucosal responses in the rectosigmoid, possibly due to lower mast cell numbers in the distal segments of the large intestine. ${ }^{13}$ In six patients, provocation was performed during gastroduodenoscopy. The mucosal reactions in the stomach were inconsistent, whereas in the duodenum the test could be performed with good results. However, gastroduodenoscopy over 20 minutes was not well tolerated by the patients. Furthermore, the provocation test is more troublesome to carry out in the duodenum for the endoscopist because its peristaltic movements interfered with the test procedure. The pro- cedure could not be completed in four patients for this reason. In five patients, the COLAP test was performed twice. At least two antigens, one of which was tested positive and one negative within the first COLAP test, were given for a second time three to 16 months later and caused almost identical results suggesting that the results of the COLAP test are reproducible (data not shown).

Antigen extracts were given by injection into the mucosa using an instrument similar to that used for endoscopic sclerotherapy of oesophageal varices (Kobi $\mathrm{GmbH}$, Lehrte, Germany). This consists of a covered plastic tube $150 \mathrm{~cm}$ long, which is connected to a fine needle at its distal end and a syringe containing the antigen extract at its proximal end. Usually, five test solutions (three antigen extracts, one negative control, one positive control), each at $250 \mu \mathrm{l}$, were given. The plastic tube (void volume of about $0.8 \mathrm{ml}$ ) was filled with the test solution before introducing it into the working channel of the endoscope to avoid contamination, the tube was drawn out of the endoscope after each application, and depleted of the test solution by washing with $4 \mathrm{ml} 0.9 \%$ sodium chloride and pressing $8 \mathrm{ml}$ air through the tube before it was filled again with the next test solution. The diluent of antigens $(0.9 \%$ sodium chloride) served as a negative control and a solution containing histamine (3.4 $\mu \mathrm{g} / \mathrm{ml}$, diluted in $0.9 \%$ sodium chloride) as a positive control. A distance of at least $3 \mathrm{~cm}$ was maintained between the injection sites. The length of the needle could be regulated externally, and was adjusted to a length of about $2 \mathrm{~mm}$ before injection. The reactions were continuously registered on a video tape. As performed in skin prick tests, the mucosal weal and flare reaction was classified semiquantitatively 20 minutes after challenge using a scale of 0 to 4: $0=$ no reaction, $1=$ questionable reaction, 2=moderate reaction $(<1 \mathrm{~cm}$ diameter), 3=strong reaction $(1-2 \mathrm{~cm})$, and $4=$ maximal reaction $(>2 \mathrm{~cm})$. The reaction was classified as positive if the grade of weal or flare reaction was $\geq 2$. The mucosal reaction was assessed by two observers, the endoscopist, who was not informed about patients history and content of the given solutions, and a second person who administered the solution. If the grades of weal and flare reaction were different between the observers, the higher grade is shown in the results. The patient was not informed which antigens were given.

\section{ALLERGENS}

The food allergens were provided by $\mathrm{Dr} H$ Löwenstein (ALK Laboratories, Horsholm, Denmark) and by Dr J Ganzer (Allergopharma, Hamburg, Germany). The extracts were lyophilised and did not contain additives such as glycerine or preservatives. They were reconstituted in $0.9 \%$ sodium chloride just before use or stored in small aliquots at $-80^{\circ} \mathrm{C}$ to avoid freeze-thaw cycles. The concentrations used for intestinal challenge were assessed by dose response 
experiments in four patients with clear evidence of food allergy as confirmed by elimination diet and rechallenge experiments. All extracts tested in these experiments induced a grade $2-4$ reaction at a 1:10 dilution of the stock solutions containing $3 \mathrm{mg} / \mathrm{ml}$ protein. Therefore, this dilution was used in all experiments. Its activity was similar to that of skin prick test solutions, as confirmed by skin tests. A total of 210 antigens (three in each patient) were tested in the COLAP test. The antigens were selected according to the patients' history of food intolerance and presence of specific IgE in serum (history, or RAST, or both were positive for 158 antigens). Fifty two antigens, for which both history and RAST were negative, have also been tested for control purposes. Healthy control subjects were challenged with a defined set of three antigens (milk, wheat, hazelnut) assumed to be of particular relevance, as these antigens often tested positive in patients.

SKIN TESTS AND LABORATORY VARIABLES In 46 out of 70 patients and in all healthy controls, skin prick tests for the most common inhalative and food allergens (ALK-Scherax, Hamburg, Germany) were performed. In all patients, specific IgE against food allergens selected according to patients' history and the skin test was measured by RAST (Pharmacia CAP system, class 0-6, Pharmacia, Uppsala, Sweden). In healthy controls, RAST for the most common food antigens (mixed antigens) was performed. In each subject, a panel of other routine serum and blood variables was measured. Eosinophil cationic protein (ECP) and eosinophil protein $\mathrm{X}$ (EPX) were measured in the serum of each person at the day of COLAP test by radioimmunoassay (RIA) (Pharmacia).

\section{IMMUNOHISTOCHEMISTRY AND ELECTRON} MICROSCOPY

Three mucosal biopsy specimens were taken 20 minutes after administration of the test solutions from each site to quantify intestinal mast cells as well as eosinophils and to study their grade of activation. Tissue sections $(n=250)$ from all subjects were stained with monoclonal antitryptase antibody (Chemikon, Temecula, CA, USA) for mast cell counting, and with monoclonal anti-ECP antibody EG2 (Kabi-Pharmacia) for eosinophil counting. The methods of tissue processing and cell counting within the intestinal lamina propria have been described in detail elsewhere. ${ }^{13}$ Eosinophil activation (using electron microscopy and immuno-electron microscopy) and mast cell degranulation (using immunohistochemistry) were studied in 10 selected patients with a pronounced macroscopic reaction (grades 3-4) of the mucosa to at least one antigen and in five healthy control subjects. For standard electron microscopy and immunogold staining, biopsy specimens were processed as previously described. ${ }^{14}$ For immunogold staining, sections were incubated for 12 hours at $4^{\circ} \mathrm{C}$ with anti-ECP antibody or control antibodies of the same isotype (Dianova, Hamburg, Germany) at different concentrations. After washing, sections were incubated with protein A-gold complex (1:20 dilution, Amersham Corp, Arlington, IL, USA) of $15 \mathrm{~nm}$ colloid gold for two hours at room temperature. Sections were finally washed and stained with uranyl acetate and lead citrate as described. ${ }^{15}$ Eosinophils were examined in the electron microscope sections for different criteria, such as loss of intact secondary granules, formation of small dense granules or vesicles in the cytoplasm, and morphological evidence for degranulation. ${ }^{14}$ According to these criteria, the grade of eosinophil activation was evaluated semiquantitatively using a scale from 0 to $3(0=$ no, $1=$ weak, $2=$ strong, $3=$ maximal activation). The observers of the tissue sections were not informed about patients' clinical and endoscopic data. Mast cell activation was examined by immunohistochemistry using an antihistamine antibody provided by F Sundler, Lund, Sweden. Tissue processing and staining were performed as described. ${ }^{16} 17$

\section{STATISTICS}

Data are presented as mean (SD) if not indicated otherwise. Means of two groups (multiple groups) were compared by one way analysis of variance (ANOVA) and $t$ test. Qualitative data were compared by Pearson's $\chi^{2}$ test. Histological data were analysed for correlation with endoscopic results by calculating Spearman's rank correlation coefficient $r_{s}$.

\section{Results}

MUCOSAL REACTION AFTER COLAP

Fifty four of 70 patients $(77 \%)$ were positive in the COLAP test - that is, one or more food antigen extract(s) induced a weal or flare reaction of grade $\geqslant 2$. Sixteen patients (one with eosinophilic enterocolitis, one with ulcerative colitis, 14 without evidence for organic disease) did not react to any of the food antigens tested. Figure 1 gives an example of a mucosal reaction in response to intestinal allergen provocation. In the group of patients, 97 out of 210 antigen extracts $(46 \%)$ induced a positive reaction (Table I). The rate of significant weal and flare reactions in response to antigens for which patients' history was positive was higher than that induced by antigens not mentioned in patients' history $(51 \% v 34 \%$, Pearsons' $\chi^{2}$ test, $\left.p=0.025\right)$. In control subjects, there were no significant reactions in response to all antigen challenges $(n=15)$. The weal and flare reaction started about two to three minutes after antigen (or histamine) application and reached a maximum after 10 to 15 minutes. The grade of reddening and swelling of the mucosa in response to antigen and histamine provocation were highly correlated $\left(r_{\mathrm{s}}=0.65, \mathrm{p}<0.001, \mathrm{n}=280\right)$, but reddening was generally less pronounced. A positive mucosal response was often accompanied by an increase 

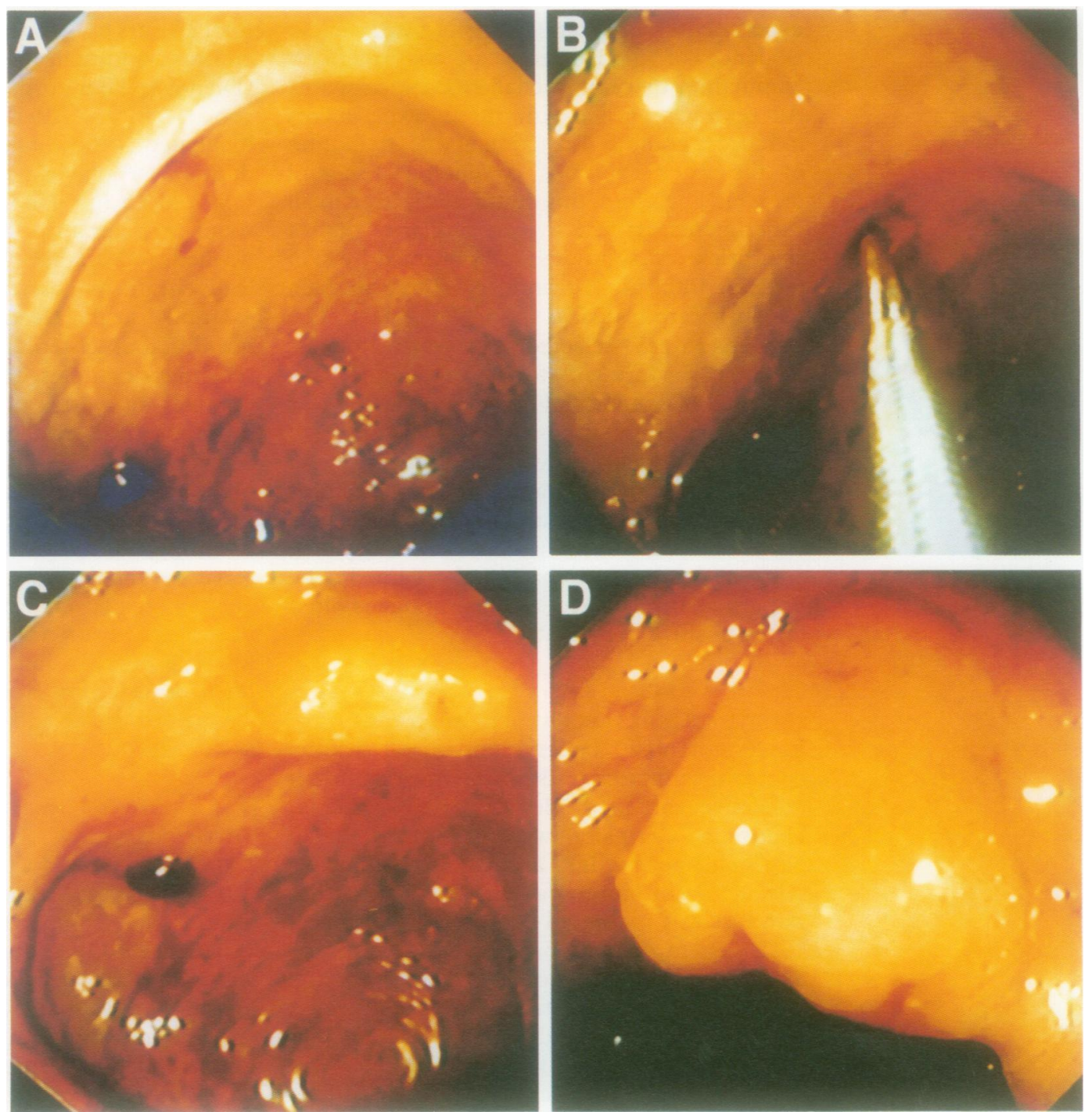

Figure 1: Mucosal reaction after endoscopic challenge of the caecal mucosa with wheat antigen. (A) normal mucosa before challenge; (B) application of the antigen extract by a fine needle; (C) mucosal reaction after three minutes; (D) mucosal reaction after 15 minutes.

in peristaltic movements of the colon. No mucosal reactions were found in patients or controls in response to buffer control. The response to histamine challenge was positive in 49 of 70 patients $(70 \%)$ and in four of five controls. In the group of patients, the weal and flare reaction to histamine was slightly more

TABLE I Mucosal reactions to different food antigens

\begin{tabular}{lllcccl}
\hline History & RAST & Test solution & Number $(n)$ & PositiveS & PositiveS & $\begin{array}{l}\text { Weal and flareS } \\
(\text { mean }(S D))\end{array}$ \\
\hline & & Buffer control & 70 & 0 & 0 & $0 \cdot 10(0 \cdot 59)^{\star}$ \\
& Histamine & 70 & 49 & 70 & $2 \cdot 17(1 \cdot 29)^{\star}$ \\
+ & Antigens (all) & 210 & 97 & 46 & $1 \cdot 34(1 \cdot 22)^{\star}$ \\
+ & + & Antigens & 19 & 10 & 53 & $1 \cdot 53(1 \cdot 22)^{\star \star}$ \\
+ & - & Antigens & 85 & 42 & 49 & $1 \cdot 46(1 \cdot 26)^{\star \star}$ \\
- & Antigens & 42 & 23 & 55 & $1 \cdot 50(1 \cdot 29)^{\star \star}$ \\
- & Antigens & 12 & 8 & 67 & $1 \cdot 67(1 \cdot 37)^{\star \star}$ \\
- & Antigens & 36 & 12 & 33 & $1 \cdot 03(1 \cdot 05)^{\star \star}$ \\
& + & Antigens & 16 & 2 & 13 & $0 \cdot 56(0 \cdot 73)^{\star \star}$ \\
& Milk & 47 & 19 & 40 & $1 \cdot 23(1 \cdot 24)$ \\
& Wheat & 37 & 17 & 46 & $1 \cdot 41(1 \cdot 28)$ \\
& Hazelnut & 19 & 10 & 53 & $1 \cdot 58(1 \cdot 02)$ \\
& Apple & 19 & 9 & 47 & $1 \cdot 53(1 \cdot 47)$ \\
& Pork & 11 & 8 & 73 & $1 \cdot 91(1 \cdot 38)$ \\
& Paprika & 10 & 4 & 40 & $1 \cdot 00(1 \cdot 15)$ \\
& Egg & 9 & 3 & 33 & $0 \cdot 78(0.97)$ \\
& Beef & 6 & 2 & 33 & $1 \cdot 17(0 \cdot 73)$ \\
& Other antigens & 52 & 25 & 48 & $1 \cdot 32(1 \cdot 24)$ \\
& & &
\end{tabular}

$\star / \star \star p<0.001$ Differences between group means (one way ANOVA).

fWeal and flare reactions were graded from 0 to 4 (see text); positive is defined as grade $\geq 2$. pronounced than in healthy controls (grade $2 \cdot 2$ $(1 \cdot 3)$ v $1.6(0 \cdot 9))$.

SYSTEMIC REACTIONS AFTER COLAP

Fatal reactions such as anaphylactic decrease in blood pressure, angio-oedema, or asthmatic attacks in response to intestinal provocation have not been seen, despite the fact that some of the patients had a history of systemic anaphylactic reactions to ingestion of particular foods. In one patient, a generalised urticaria was provoked by the test, and occurred about 20 minutes after intestinal antigen administration. This patient had a history of abdominal symptoms and urticaria after eating fish, he had specific IgE (CAP class 2 ) but a negative skin test against trout, and his intestinal mucosa was challenged with milk (negative history, COLAP response grade 1), wheat (unclear history, COLAP response grade 3 ), and trout (COLAP response grade 3) extract. A few other patients (six with positive results in the COLAP test, two with negative results) indicated mild skin reactions such as itching or gastrointestinal symptoms - for example, cramps or diarrhoea - within the first 24 hours 
after intestinal provocation. The symptoms of the two patients without mucosal response in the COLAP test may be due to "late phase reactions" typically occurring four to eight hours after antigen contact, which cannot be registered by the COLAP test. Most patients did not experience any symptoms after the COLAP test.

\section{RELATION BETWEEN COLAP TEST AND OTHER} CLINICAL OR LABORATORY DATA

Skin prick tests were performed on 46 patients. Six patients were negative for the most common food antigens. In total, 410 out of 540 skin prick tests $(76 \%)$ for food antigens were negative, and the rate of negative results was hardly reduced by testing with native foods as performed occasionally in doubtful cases. No significant relation between results from skin tests and COLAP test $(n=56)$ or between skin tests and history of food intolerance $(n=56)$ were found (Pearson's $\chi^{2}$ test, both $p>0 \cdot 1$ ). For almost half of the antigens, results of the COLAP test and skin test corresponded (14\% both positive, $30 \%$ both negative). However, $11 \%$ of the antigens were tested positive in the skin test and negative in the COLAP test, and $45 \%$ of the antigens induced a weal and flare reaction in the intestine but not in the skin. Most antigens (21 out of 25 ) that tested negative in the skin test but positive in the

TABLE II Laboratory findings (mean (SD))

\begin{tabular}{llll}
\hline & $\begin{array}{l}\text { Healthy } \\
\text { controls } \\
(n=5)\end{array}$ & $\begin{array}{l}\text { Patients with } \\
\text { negative COLAP } \\
(n=16)\end{array}$ & $\begin{array}{l}\text { Patients with } \\
\text { positive COLAP } \\
(n=54)\end{array}$ \\
\hline Lariable & $8 \cdot 4(4 \cdot 1)$ & $6 \cdot 7(2 \cdot 7)$ & $7 \cdot 7(2 \cdot 6)$ \\
Eoucocytes $\left(\times 10^{3} / \mu \mathrm{l}\right)$ & $2 \cdot 2(1 \cdot 8)$ & $3 \cdot 5(3 \cdot 1)$ & $4 \cdot 7(6 \cdot 2)$ \\
IgE (units/ml) & $71(63)$ & $60(50)$ & $360(609)$ \\
Eosinophil cationic protein $(\mu \mathrm{g} / \mathrm{l})$ & $6 \cdot 4(5 \cdot 1)$ & $9 \cdot 6(8 \cdot 8)$ & $13 \cdot 4(15 \cdot 4)$ \\
Eosinophil protein X $(\mu \mathrm{g} / 1)$ & $14 \cdot 4(5 \cdot 8)$ & $29 \cdot 0(15 \cdot 6)$ & $37 \cdot 9(46 \cdot 7)$ \\
Intestinal mast cells $(\%$ of lamina propria cells) & $2 \cdot 1(1 \cdot 9)$ & $2 \cdot 3(1 \cdot 6)$ & $2 \cdot 3(1 \cdot 5)$ \\
Intestinal eosinophils $(\%$ of lamina propria cells) & $7 \cdot 7(5 \cdot 7)$ & $9 \cdot 8(9 \cdot 3)$ & $5 \cdot 5(2 \cdot 4)$ \\
\hline
\end{tabular}

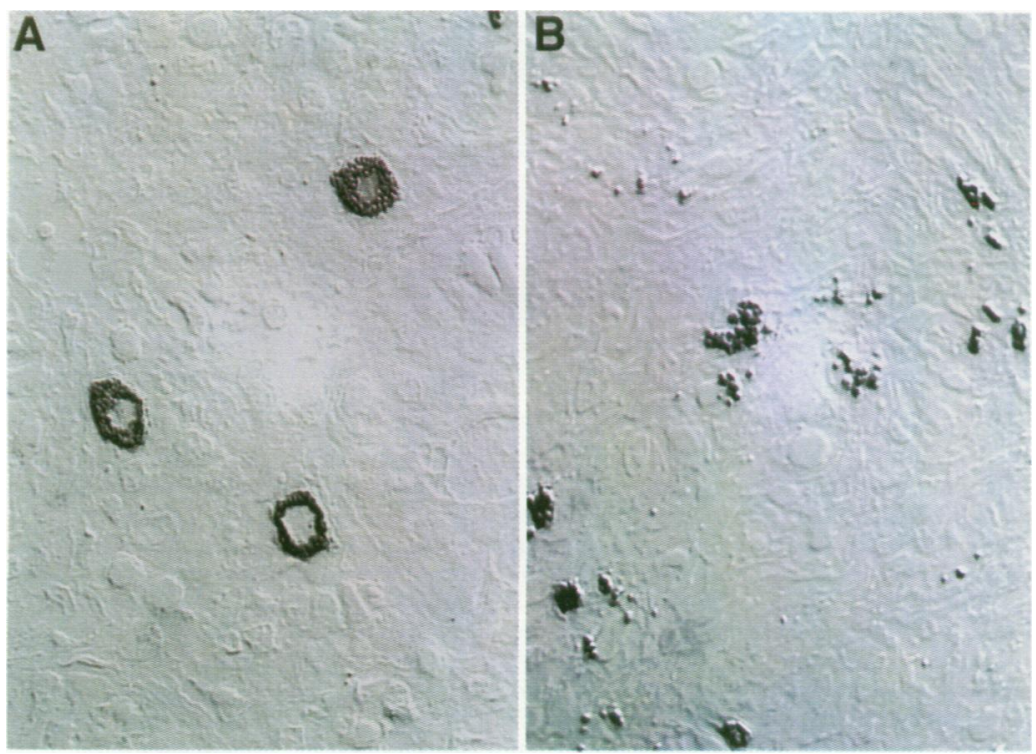

Figure 2: Mast cell activation after intestinal allergen provocation. Immunohistochemical staining of histamine in tissue sections of caecal mucosa removed 20 minutes after challenge with buffer control $(A)$ or milk antigen extract $(B)$. The buffer control induced no macroscopic changes of the mucosa; the milk extract induced a significant weal and flare reaction (grade 3). Magnification originally $\times 400$.
COLAP test induced adverse reactions according to patients' history. Specific IgE against at least one food antigen could be detected in 49 out of 64 patients $(77 \%)$, albeit at very low levels in most cases (in 38 patients, the maximal RAST class was 2 , in 11 patients, the maximal RAST class was 3 or 4, PharmaciaCAP-system). In total, 696 RASTs were performed in the patient group, $129(19 \%)$ of which were positive $(\geq 2)$. The results of the RASTs were not correlated with patients' history or with the COLAP test (Pearson's $\chi^{2}$ test, both $\mathrm{p}>0 \cdot 1)$. Other laboratory variables indicating intestinal inflammation (blood sedimentation rate, leucocytes, $\mathrm{C}$ reactive protein) or related to allergic disease (total serum IgE, eosinophil count, eosinophil granule proteins) were slightly raised in the group of patients with a positive COLAP test, albeit not significantly compared with patients with a negative COLAP test and healthy controls (ANOVA, $p>0.05$; Table II).

Comparing the patients a with negative and positive COLAP test, no significant differences between the frequency of a history of food intolerance $(81 \% v 89 \%)$, of atopic diseases such as seasonal rhinitis, extrinsic asthma, or atopic dermatitis $(44 \% v 59 \%)$, or of abdominal symptoms such as diarrhoea $(56 \% v$ $69 \%$ ), abdominal pain $(63 \%$ v $43 \%)$, or flatulence $(44 \% v 22 \%)$ were found. The results of the COLAP test in patients with intestinal inflammation ( $88 \%$ positive, $n=17)$ were not significantly different from those without such disease $\left(74 \%\right.$ positive, $n=53, \chi^{2}$ test, $p>0 \cdot 1)$. In patients who received premedication with pethidine and midazolam, the mucosal reactions were slightly reduced (in response to antigen $1.3(1.3)$, in response to histamine $2 \cdot 1(1 \cdot 3), \mathrm{n}=54)$ compared with untreated patients (in response to antigen 1.5 $(1 \cdot 1)$, in response to histamine $2.6(1 \cdot 3)$, $\mathrm{n}=16)$. The difference was not significant (Mann-Whitney U test, $p>0 \cdot 1$ ). Sixty seven per cent of the patients who had no clear history of food intolerance but a positive RAST or skin test for food antigens ( $n=9)$, had at least one positive reaction in the COLAP test, compared with $79 \%$ of patients with food intolerances $(n=61)$. This difference was not significant.

\section{ACTIVATION OF MUCOSAL EOSINOPHILS AND} MAST CELIS

The number of mast cells and eosinophils, expressed as a percentage of lamina propria cells (Table II), was not changed by challenge with allergen extracts or histamine compared with control tissue in which diluent was injected. However, immunohistochemical studies indicate that mast cells degranulate in response to allergens which induced a significant mucosal reaction. In all tissue specimens derived from mucosal sites where antigens induced a weal and flare reaction of grade 3 or 4, a pronounced loss of cellular granules was seen (Fig 2). By contrast, no mast cell changes were seen in response to negative control solution and to histamine application. Allergens which failed to induce a weal and flare reaction 

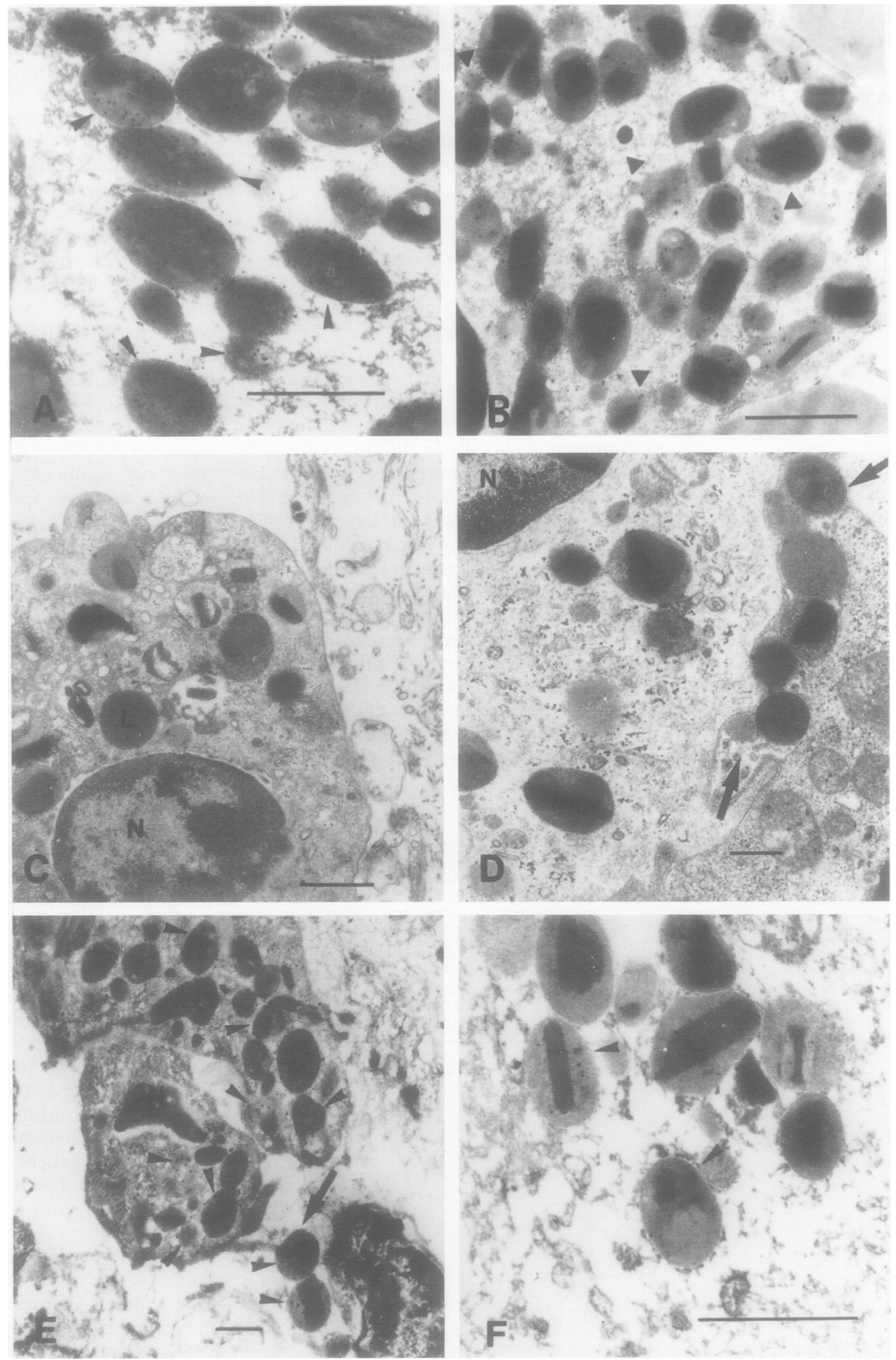

Figure 3: Eosinophil activation after intestinal allergen provocation. Mucosal biopsy specimens were processed for electron microscopy and immunogold staining with an antibody against eosinophil cationic protein (ECP) as described in the methods section. (A) negative control. There are no signs of eosinophil activation, no small dense granules, no vesicles, granule matrix is intact, arrow heads show gold particles indicating position of ECP in the matrix but not in the core of the secondary granules; (B) challenge with pork extract, mucosal reaction grade 3. Arrow heads show gold particles in the matrix of intact secondary granules and in small dense vesicles, which are newly formed in activated eosinophils; $(C)$ challenge with histamine, mucosal reaction grade 4 (highly activated eosinophils, newly formed vesicles in the cytoplasm and in the matrix of the secondary granules, loss of matrix content. $N=$ nucleus; $L=$ lipid bodies which are typically found in activated eosinophils); (D) challenge with histamine, mucosal reaction grade 4. (Arrows show granules with matrix and core outside of the eosinophils; clear signs of degranulation accompanied by a decreased number of granules within the cytoplasm can be seen regularly in grade 4 reactions); (E) challenge with hazelnut extract, mucosal reaction grade 3. There is a change in shape of eosinophils, with irregular cellular surfaces. Arrows show degranulation; arrowheads show gold particles located in the matrix of secondary granules and in newly formed small dense granules; $(F)$ challenge with hazelnut, mucosal reaction grade 3. Arrow heads show released secondary granules that also contain gold particles in their matrix. Bars indicate $1 \mu \mathrm{m}$. 
of the mucosa (grade 0-1) also failed to cause significant mast cell activation. Furthermore, we found a strong activation of lamina propria eosinophils at sites of antigen induced weal and flare reactions (Fig 3). Electron microscopy studies showed that eosinophils (by contrast with mast cells) became activated and degranulated not only by particular food antigens but also by histamine. The extent of weal and flare reaction of the mucosa and eosinophil activation was positively correlated (Fig 4). In healthy control subjects who underwent the COLAP test, virtually no mast cell and eosinophil activation was seen (data not shown).

\section{FOLLOW UP OF THE PATIENTS}

All patients who showed at least one positive reaction in the COLAP test were given an antiallergic treatment. Reliable data concerning the clinical follow up over a period of at least six months after a COLAP test were available from 35 of 54 patients with at least one positive result in the COLAP test. Information was derived from a detailed questionnaire. The basic treatment in all patients was an individual diet with strict elimination of each food that tested positive in the COLAP test. All 35 questioned patients followed the recommendations of the dietician and kept to this diet. The results of the COLAP test
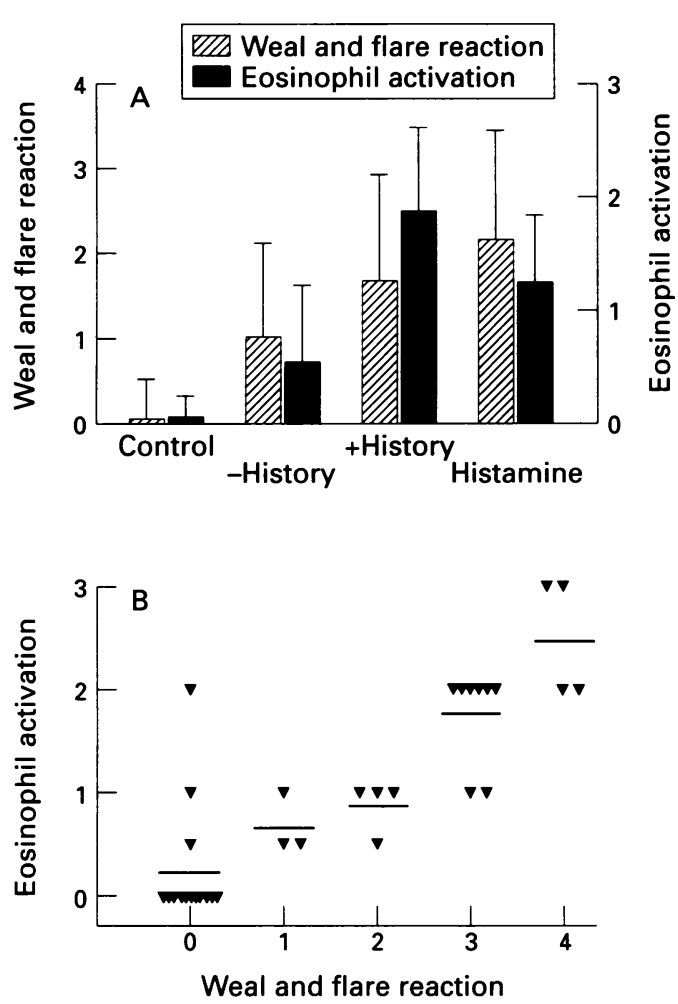

Figure 4: Relation between weal and flare reaction and eosinophil activation after intestinal allergen provocation. ( $A$ ) Eosinophil activation (grade 0-3; black columns) in response to intestinal challenge with $0.9 \%$ sodium chloride (control), food antigen with negative $(-)$ or positive (+) history of food intolerance, and histamine $(3.4 \mu \mathrm{g} / \mathrm{ml})$ in 10 patients (mean (SD)). For comparison, mucosal weal and flare reactions (grade 0-4; hatched columns) in 70 patients are shown. (B) Correlation between macroscopic weal and flare reaction and microscopic eosinophil activation (Spearman's rank correlation coefficient $\left.r_{s}=0.86, n=33, p<0 \cdot 0001\right)$. induced a change in nutrition habits in 21 patients. Fourteen patients had already eliminated the positive food on suspicion before the COLAP test; its results confirmed their diet as efficient. Nine of 35 patients had to be treated with additional antiallergic medication (disodium cromoglycate, oral dose $4 \times 200 \mathrm{mg}$ / day; and steroids, oral dose $7.5 \mathrm{mg} /$ day in two patients). A consistent improvement of symptoms was achieved in 29 of 35 patients $(83 \%)$, according to the patient's global assessment and objective assessments such as endoscopy and laboratory changes. In six patients, previous treatment with aminosalicylates or corticosteroids could be definitively terminated (data not shown).

\section{Discussion}

Our study shows that endoscopic challenge of the human intestinal mucosa with particular food antigen extracts can induce a weal and flare reaction in selected patients with unclear abdominal symptoms or intestinal inflammation and a history of food intolerance. The data provide evidence that the reactions of the COLAP test are specific. Firstly, the test was positive in $77 \%$ of selected patients with suspected intestinal food allergy but in none of the control subjects without signs of allergic disease. Secondly, in the patients a mucosal reaction only occurred in response to $46 \%$ of all food challenges making it unlikely that food antigens generally induce a non-specific mucosal reaction. Thirdly, the buffer control solution always failed to induce a mucosal reaction, whereas application of histamine caused a weal and flare reaction in almost all patients, largely excluding "false negative responses" due to technical reasons. Fourthly, the mucosal response towards food antigens was related to the patients' history.

It may be questioned whether the duodenum is more suitable for testing food allergy than the proximal colon, as macromolecules are more likely to be taken up in the small intestine. However, the site of absorption of food proteins is not exactly known and may differ between patients. Furthermore, our study shows that the colonic mucosa can react to challenge with food antigen, even in patients with epigastric pain and vomiting. In addition, many patients had symptoms related to the distal gastrointestinal tract such as abdominal cramps and diarrhoea. Finally, the colon was chosen as an optimal site for local provocation for the technical reasons discussed in the methods section. Interestingly, the COLAP test results were somewhat weaker in patients pretreated with pethidine and midazolam before colonoscopy, compared with untreated patients. It has been reported that benzodiazepines may inhibit serotonin release in rat mast cells, ${ }^{18}$ whereas more recent studies indicate that premedication with diazepam and pethidine does not affect mast cell mediator release. ${ }^{19}$ The reason why patients had a more prominent response to histamine than control subjects is unknown. Perhaps this phenomenon is comparable with the non-specific 
bronchial hyper-responsiveness to histamine in patients with asthma. It is well known that asthmatic patients show an increased reactivity of the bronchial airways to histamine compared with controls, as measured by spirometry. ${ }^{20}$

The immunohistochemical results suggest that the mucosal response is based on an allergic reaction, as two major effector cells of allergic inflammation, mast cells and eosinophils, became activated by antigen challenge in the COLAP test. The macroscopic reaction of the mucosa in response to antigen challenge was strongly correlated with intestinal mast cell and eosinophil activation, a finding that further argues against the possibility of a non-specific mucosal reaction in response to endoscopic challenges. The role of mast cells in immediate type allergic reactions and the mechanism of antigen dependent mast cell activation by crosslinking surface bound IgE is well established. By contrast, eosinophils are suspected to be involved in the late phase rather than the early phase of allergic reactions. ${ }^{21} 22$ In the COLAP test, we saw a rapid (within 20 minutes) activation of intestinal eosinophils in vivo caused by food antigens, which has not been reported before. The mechanism of this eosinophil activation is unclear at present. It is known that eosinophils can be activated by $\operatorname{IgE}$ independent triggers, but not by allergens, although eosinophils bear IgE binding sites, the functional role of which is not clear. ${ }^{23-25}$ In vivo studies have shown that intestinal eosinophils become activated in the course of diseases such as eosinophilic enteritis, coeliac disease, and Crohn's disease, which may indicate that as yet undefined antigens and cofactors are capable of activating intestinal eosinophils. ${ }^{26} 27$ On the other hand, eosinophil activation may occur indirectly in response to cell derived mediators - for example, from mast cells such as histamine, platelet activating factor, or interleukin-5 - known to regulate eosinophilic function..$^{23}$ In particular, the release of histamine may be a relevant trigger of intestinal eosinophils, as histamine caused an activation of intestinal eosinophils in the COLAP test.

The mechanism of intestinal food allergy seems to be different from classic type I hypersensitivity reactions of the skin or the respiratory mucosa, as skin tests were often negative and specific IgE in serum was rather low for the food antigens which caused intestinal weal and flare reactions. On the other hand, the COLAP test was mostly negative for antigens tested positive in skin tests or RAST, but for which no adverse reaction was described by the patient. These findings suggest that in patients with gastrointestinal disease, clinically relevant food antigens can be identified by the COLAP test, but not by skin test and measurement of specific IgE. To explain the finding that patients' history, but not specific IgE or skin reactions were correlated with the results of the COLAP test, two hypotheses may be envisaged, which are not mutually exclusive. Either specific IgE is present only in the intestinal mucosa but not systemically, or intestinal reactions are not (or not only) mediated by an IgE dependent mechanism. Several studies have suggested that mechanisms other than IgE mediated reactions may be involved in the pathogenesis of food allergy. ${ }^{29} 30$

The double blind placebo controlled oral food challenge test and the methods of elimination diet and rechallenge have been proposed as "gold standards" for the confirmation of food allergy. ${ }^{31}$ However, these tests are often not pragmatic in practice. In particular, for gastrointestinal manifestations, their interpretation is difficult, because the readout system depends on the patients' symptoms. In addition, the time course of occurrence of gastrointestinal symptoms after oral food challenge is highly variable. ${ }^{36}$ The COLAP test has several advantages to oral food challenge procedures, such as the low amounts of allergen required to induce a response (which reduces the possibility of fatal reactions), the shortness of the test yielding results within a few minutes (making is possible to perform the test on an outpatient basis), and the objectiveness of the readout system (which is also performed in a double blind placebo controlled fashion). The fact that severe systemic reactions were not found in patients who underwent intestinal provocation suggests that the COLAP test is safe, although a setting prepared for emergency situations is recommended when performing this test. Due to the lack of reliable and pragmatic diagnostic means to confirm the diagnosis of intestinal food allergy, the positive and negative predictive values of the COLAP test cannot be calculated at present. The previously presented antigen challenge procedures performed in the stomach had the same deficiency. ${ }^{9-12}$ Only the follow up of patients with positive results in the COLAP test treated accordingly with elimination diet or antiallergic drugs will allow an estimation of the definitive clinical relevance of mucosal provocation tests.

Fifty three of the patients examined in this study had abdominal symptoms without evidence of intestinal inflammation or other organic disorders, and 39 of them were positive in the COLAP test, supporting the hypothesis that allergic reactions may play a part in a subgroup of patients with irritable bowel syndrome. ${ }^{32}$ According to our preliminary follow up data, the COLAP test may improve the clinical management of such patients with "non-organic" abdominal symptoms and suspected intestinal food allergy. In addition, it has been suggested that mechanisms of allergic inflammation may be related to inflammatory bowel conditions of unclear origin such as eosinophilic enteritis, coeliac disease, Crohn's disease, and ulcerative colitis. ${ }^{33-35}$ We found that 15 out of 17 patients with different kinds of inflammatory bowel conditions were positive in the COLAP test. Thus the COLAP test may be of clinical relevance also in a selected subgroup of patients with inflammatory bowel conditions. In conclusion, this study provides evidence supporting this "intestinal prick test" as a valuable diagnostic tool and useful for the study of mechanisms of gastrointestinal food allergy. 
Parts of this study were supported by grants of the Else-KrönerStiftung, Bad Homburg, Germany, and of the Deutsche Forschungsgemeinschaft, Bonn, Germany (SFB280-C8). We thank Ms Gisela Weier and Ms Kathrin Wordelmann for their expert technical assistance, and the staff of the endoscopy unit expert technical assistance, and the staff of the endoscopy unit
for excellent cooperation. Parts of this study have been for excellent cooperation. Parts of this study have been
presented (and published in abstract form) at the 95th Annual presented (and published in abstract form) at the 95th Annual
meeting of the American Gastroenterological Association, San meeting of the American Gastroenterological Association, San Diego 1995 (Gastroenterology 1995; 108: A784), the 8th International Congress of Mucosal Immunology, San Diego United European Gastroenterology Week, Berlin 1995 (Gut 1995; 37 (suppl 2): A99).

1 Farah DA, Calder I, Benson L, Mackenzie JF. Specific food intolerances: its place as a cause of gastrointestinal sympintolerances: its place as a caus

2 Ferguson A. Food sensitivity or self-deception? $N$ Engl $\mathcal{F}$ Med 1990; 323: 476-8.

3 Crowe SE, Perdue MH. Gastrointestinal food hypersensitivity: basic mechanisms of pathophysiology. Gastroenterology 1992; 103: 1075-95.

4 Young E, Stoneham MD, Petruckevitch A, Barton J, Rona R. A population study of food intolerance. Lancet $1994 ; 343$ : $1127-30$.

5 Bruijnzeel-Koomen C, Ortolani C, Aas K, Bindslev-Jensen C Björktstén B, Moneret-Vautrin D, Wüthrich B. Adverse reactions to food. Allergy 1995; 50: 623-35.

6 Food intolerance and food aversion. A joint report of the Royal College of Physicians and the British Nutrition Foundation. $₹ R$ Coll Physicians Lond 1984; 18: 83-123.

7 Freed DLJ. Laboratory diagnosis of food intolerance. In Brostoff J, Challacombe SJ, eds. Food allergy and intolerance. London: WB Saunders, 1987: 873-97.

8 Jewett DL, Fein G, Greenberg MH. A double-blind study of symptom provocation to determine food sensitivity. $N$ Engl f Med 1990; 323: 429-33.

9 Pollard HM, Stuart GJ. Experimental reproduction of gastric allergy in human beings with controlled observations on the mucosa. 7 Allergy 1942; 13: 467-73.

10 Romanski $B$. The pathology of food allergy studied by gastric allergen challenge. In: Brostoff J, Challacombe SJ, gastric allergen challenge. In: Brostoff J, Challacombe S J, 1987: 917-31.

11 Reimann HJ, Lewin J. Gastric mucosal reactions in patients with food allergy. Am f Gastroenterol 1988; 11: 1212-9.

12 Bagnato GF, Di Cesare E, Caruso RA, Gulli S, Cugliari A Morabito Lo Prete A, et al. Gastric mucosal mast cells in atopic subjects. Allergy 1995; 50: 322-7.

13 Bischoff SC, Wedemeyer J, Herrmann A, Meier PN, Trautwein C, Cetin Y, et al. Quantitative assessment of intestinal eosinophils and mast cells in inflammatory bowel disease. Histopathology 1996; 28: 1-13.

14 Zeck-Kapp G, Czech W, Kapp A. TNF+-induced activation of eosinophil oxidative metabolism and vation of eosinophil oxidative metabolism and morphology

15 Milici AJ, Porter GA. Lectin and immunolabeling of microvascular endothelia. F Electron Microsc Tech 1991; 19: 305-15.

16 Cetin Y, Grube D. Immunoreactives for chromogranin A and $\mathrm{B}$, and secretogranin II in the guinea pig enteroendocrine system: cellular distributions and intercellular heterogeneities. Cell Tissue Res 1991; 264: 231-41.

17 Panula P, Häppölä O, Airaksinen NS, Auvinen S, Virkamäki A. Carbodiimide as a tissue fixative histamine immunohistochemistry and its application in developmental neurobiology. 7 Histochem Cytochem 1988; 36: 259-69.

18 Suzuki-Nishimura $T$, Sano $T$, Uchida MK. Effects of benzodiazepines on serotonin release from rat mast cells. Eur 7 Pharmacol 1989; 167: 75-85.

19 Raithel M, Hochberger J, Hahn EG. Effect of colonoscopy premedication containing diazepam and pethidine on the release of mast cell mediators in gut mucosal samples. Endoscopy 1995; 27: 415-23.

20 Cockcroft DW, Killian DN, Mellon JJA, Hargreave FE. Bronchial reactivity to inhaled histamine: a method and clinical survey. Clin Allergy 1977; 7: 235-43.

21 Charlesworth EN, Hood AF, Soter NA, Kagey-Sobotka A Norman PS, Lichtenstein LM. Cutaneous late-phase response to allergen. Mediator release and inflammatory cell infiltration. $\mathcal{f}$ Clin Invest 1989; 83: 1519-26.

22 Bousquet J, Chanez P, Lacoste JY, Barneon G, Ghavanian N, Enander I, et al. Eosinophilic inflammation in asthma. $N$ Engl ₹ Med 1990; 323: 1033-9.

23 Takafuji S, Bischoff SC, De Weck AL, Dahinden CA. IL-3 and IL-5 prime normal human eosinophils to produce leukotriene $\mathrm{C} 4$ in response to soluble agonists. $\mathcal{F}$ Immunol 1991; 147: 3855-61

24 Soussi Gounni A, Lamkhioued B, Delaporte E, Dubost A Kinet JP, Capron A, Capron M. The high-affinity IgE receptor on eosinophils: from allergy to parasites or from parasites to allergy? f Allergy Clin Immunol 1994; 94: 1214-6.

25 Bjerke $T$, Nielsen $S$, Kochen J, Rudinger N, Nielsen LP, Schiotz PO, et al. IgE receptor a-subunit is synthesized and prestored in human eosinophils [abstract]. Allergy and Clinical Immunology News 1994; (suppl 2): 80 .

26 Talley NJ, Kephart GM, McGovern TW, Carpenter HA Gleich GJ. Deposition of eosinophil granule major basic protein in eosinophilic gastroenteritis and celiac disease. Gastroenterology 1992; 103: 137-45.

27 Dvorak AM. Ultrastructural evidence for release of major basic protein-containing crystalline cores of eosinophil ranules in vivo: cytotoxic potential in Crohn's disease. fImmunol 1980; 125: 460-2.

28 Raible DG, Schulman ES, DiMuzio J, Cardillo R, Post TJ. Mast cell mediators prostaglandin-D, and histamine activast cell mediators prostaglandin-D ${ }_{2}$ and histamine acti-

29 Halpern GM, Scott JR. Non-IgE antibody mediated mechanisms in food allergy. Ann Allergy 1987; 58: 14-27.

30 Sampson HA, Broadbent KR, Bernhisel-Broadbent J. Spontaneous release of histamine from basophils and histamine-releasing factor in patients with atopic dermatitis and food hypersensitivity. N Engl $\mathcal{F}$ Med 1989; 321: 228-32.

31 Bock SA. Food challenges in the diagnosis of food hypersensitivity. In: De Weck AL, Sampson HA, eds. Intestinal immunology and food allergy. New York: Raven Press, 1995: 105-17.

32 Jones VA, McLaughlan $P$, Shorthouse $M$, Workman E Hunter JO. Food intolerance: a major factor in the pathogenesis of irritable bowel syndrome. Lancet 1982; ii: pathogenesi

33 Alun Jones V, Dickinson RJ, Workman E, Wilson AJ, Freeman AH, Hunter JO. Crohn's disease: maintenance of remission by diet. Lancet 1985 ; ii: $177-80$.

34 Marsh MN, Hinde J. Inflammatory component of celiac sprue mucosa. I. Mast cells, basophils, and eosinophils. Gastroenterology 1985; 89: 92-101.

35 Naylor AR. Eosinophilic gastroenteritis. Scott Med f 1990 35: $163-5$ 